\title{
Surgery for Spinal Trauma: Early Days at a Young Institution in a Hilly State
}

\author{
Aditya Patil ${ }^{1} \quad$ Nishant Goyal ${ }^{1} \quad$ Garga Basu ${ }^{1} \quad$ Rajnish Kumar Arora ${ }^{1} \quad$ Jitender Chaturvedi ${ }^{1} \quad$ Priyanka Gupta $^{2}$ \\ ${ }^{1}$ Department of Neurosurgery, All India Institute of Medical \\ Sciences, Rishikesh, Uttarakhand, India \\ 2Department of Anesthesia, All India Institute of Medical Sciences, \\ Rishikesh, Uttarakhand, India \\ Address for correspondence Nishant Goyal, MCh, Department
of Neurosurgery, All India Institute of Medical Sciences,
Veerbhadra Road, Rishikesh, Uttarakhand 249203, India
(e-mail: drnishantgoyal@gmail.com).
}

Indian J Neurotrauma:2020;17:90-94

\begin{abstract}
Keywords

- spinal trauma

- mode of injury

- morphology

- ASIA grade

- outcome

Background Spinal trauma is associated with significant morbidity and affects the overall quality of life of the patient as well as their family. The aim of this study was to study the epidemiology, in-hospital outcome, and follow-up of patients undergoing surgery for spinal trauma.

Methods All patients who underwent surgery for spinal trauma at the Department of Neurosurgery between June 2016 and October 2019 were studied retrospectively. Data collected from patient record included demographic profile, mode of injury, level of injury, neurological status at admission, time from injury to operative intervention, hospital stay, neurological outcome at discharge, and follow-up. Postoperative computed tomography scans were done in all patients to check for adequacy of screw placement.

Results A total of 70 patients (45 males, 25 females) with a mean age of 36 years (range: 16-65 years) were operated for spinal injuries at our department during the study period. Fall was the most common mode of injury (77.1\%), followed by road traffic accident (28.5\%). Most common site of injury was lumbar spine (38.6\%), followed by dorsal (32.9\%) and cervical (28.5\%). Out of the total, $27.1 \%$ of our patients were American Spinal Injury Association (ASIA) grade A at presentation whereas 18.6\% were ASIA E. Remaining 54.3\% patients had incomplete injuries (ASIA B, C, and D). Burst fracture was the most common morphology of injury $(60 \%)$, followed by translational injuries (30\%). Mean duration between injury and operative intervention was 20.8 days. The mean duration of hospital stay was 21.4 days (range: 8-90 days). Six patients expired during hospitalization. All these six cases had cervical spinal injury. One patient of dorsal spine injury with a complete neurological injury expired 6 months after discharge from the hospital (cause unknown). Nine of our cases showed neurological improvement on follow-up and in rest of the patients, neurological status remained same as preoperative status.

Conclusion Neurological status at admission (ASIA grade) and level of injury remain the most important predictor of the outcome. Spinal injury patients must be managed with a holistic and multidisciplinary approach. Rehabilitation is important for helping these patients in leading a productive life.
\end{abstract}

DOI https://doi.org/

10.1055/s-0040-1713330

ISSN 0973-0508.
(C)2020 Neurotrauma Society of India
License terms

(우 (1) $\Theta \circledast$ 


\section{Introduction}

Spinal cord injuries (SCIs)encompass a great deal of misery to the patients and pose a burden to the family due to the morbidity and disability associated with them. The incidence of SCI varies from 9.2 to 56.1 per million according to various epidemiological studies in different parts of the world. ${ }^{1}$ As per the report of the International Conference [Spinal Injuries Management, New Delhi (1995)], the incidence of spinal injury in India was estimated at 15 per million per year. ${ }^{2}$ In India, financial constraints and patients not reaching definitive institution are the most important factors hindering proper management of these patients. The authors aim to study the demographic profile, level of spinal trauma, in-hospital outcome, and follow-up of patients who were operated for the spinal injury at our institute.

\section{Materials and Methods}

All the patients who underwent surgery for spinal injury in the Department of Neurosurgery at All India Institute of Medical Sciences (AIIMS), Rishikesh, between June 2016 and October 2019, were included in this study. Patients were characterized according to their age, gender, mode of injury, and neurological status at admission. All the patients underwent preoperative computed tomography (CT) scan and magnetic resonance imaging (MRI) of whole spine. The preoperative imaging was studied to assess the level of involvement and morphology of injury.

All patients underwent spinal fixation under fluoroscopic guidance. The duration from time of injury to surgical intervention, level of spinal fixation done, postoperative neurological status, and duration of hospital stay were studied. All patients underwent postoperative CT scan. The neurological outcome was graded according to the American Spinal Injury Association (ASIA) grade at both admission and discharge. The in-hospital outcome and follow-up of the patients were analyzed.

\section{Results}

Over the study period, 70 patients ( 45 males and 25 females) with a mean age of 36 years (range: 16-65 years) underwent surgery for spinal injury at the Department of Neurosurgery in our institute. Patients were divided into three age categories-16 to 30 years (27 patients, group 1), 31 to 50 (33 patients, group 2 ), and 51 to 65 (10 patients, group 3)-to compare the age with mode of injury, level of injury, and neurological outcome.

In our study, fall from height was the most common mode of injury accounting for 54 cases (77.1\%), followed by road traffic accident in 16 cases (22.9\%). Of all the operated cases, 20 cases (28.5\%) involved the cervical spine (3 were of upper cervical and 17 were of subaxial cervical spine involvement), 23 cases (32.9\%) involved dorsal spine, and the lumbar spine was involved in 27 cases (38.6\%). Cervical involvement was seen in 3 patients in group 1, 13 patients in group 2, and 4 patients in group 3. Dorsal involvement was seen in 8 patients in group 1,13 patients in group 2 , and 2 patients in group 3. Lumbar involvement was seen in 15 patients in group 1,8 patients in group 2, and 4 patients in group 3 (-Fig. 1).

Neurological assessment at admission revealed that 19 patients (27.1\%) had complete SCI (ASIA grade A), $13(18.6 \%)$ were neurologically intact (ASIA grade E), while remaining 38 patients (54.3\%) had incomplete SCI (ASIA grade $B, C, D$ ). In group 1, 6 patients belonged to ASIA grade A, 6 patients belonged to ASIA grade E, while 15 were incomplete injuries. In group 2, 12 patients belonged to ASIA grade A, 2 patients belonged to ASIA grade $\mathrm{E}$, while 19 sustained incomplete cord injury. In group 3, one patient belonged to ASIA grade A, five patients belonged to ASIA grade $\mathrm{E}$, while four had incomplete injury (-Fig. 2).

In cervical injury patients, 5 patients were in ASIA A, 4 were in ASIA E, and 11 were incomplete spinal injury. In dorsal spine injury, 12 were in ASIA A, 3 were in ASIA E, and 8 were incomplete injury. In lumbar spine injury, 2 were in ASIA A, 6 were in ASIA E, and 19 were incomplete ( - Fig. 3 ).

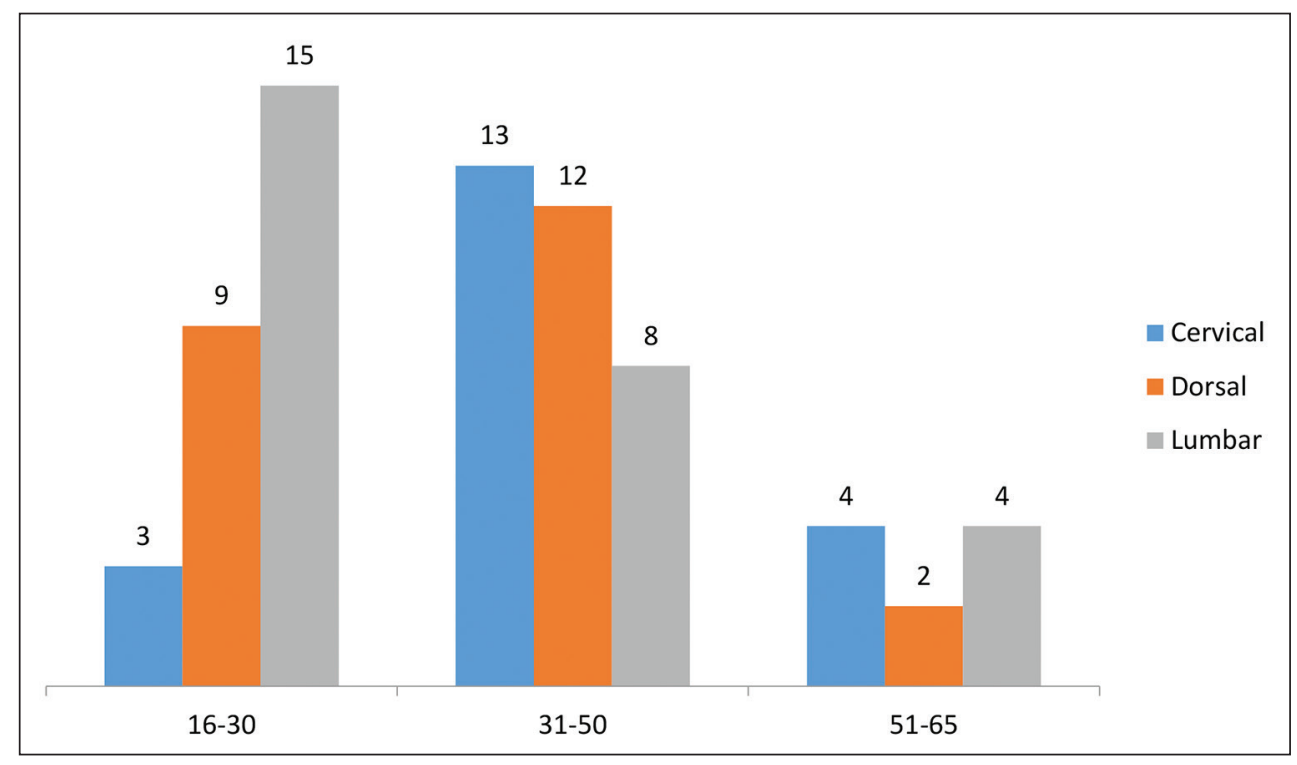

Fig. 1 Age distribution of spinal level of involvement. 


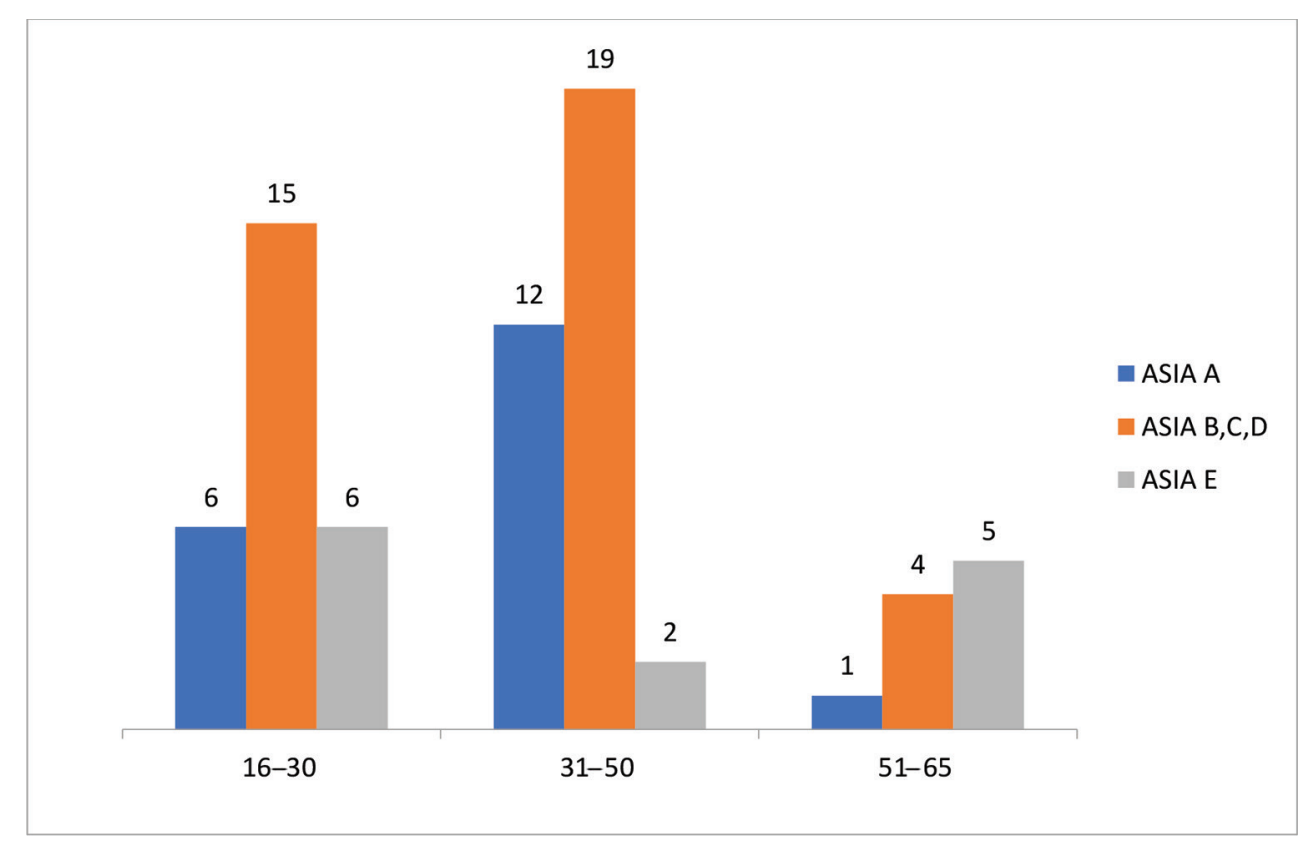

Fig. 2 Age-wise distribution according to the American Spinal Injury Association (ASIA) grade.

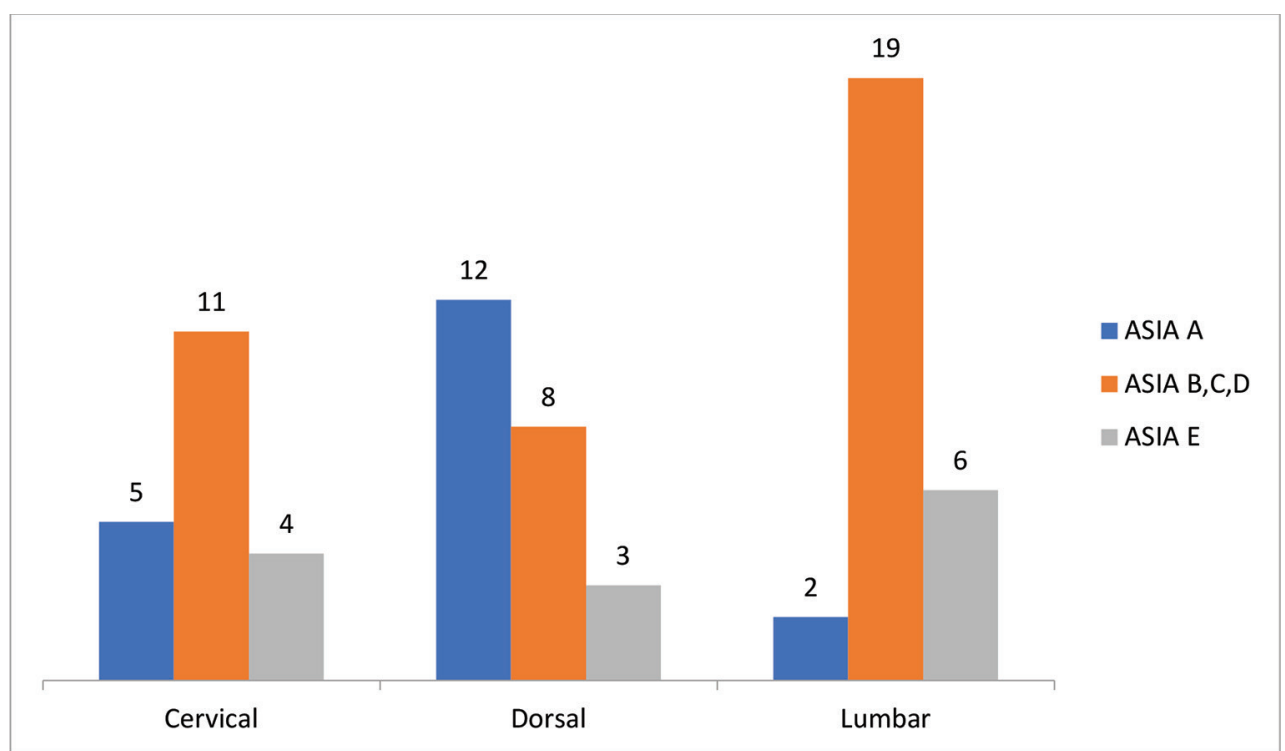

Fig. 3 Correlation of American Spinal Injury Association (ASIA) grade with level of injury.

On assessment of morphology on radiology, 42 cases were compression burst (60\%), 1 was compression fracture, 1 was distraction fracture, and 21 (30\%) were translational injuries. Three patients had upper cervical injuries and details regarding morphology were not available for two patients.

Apart from one patient who was operated 360 days after the injury, the mean duration between the time of injury and the time of surgery was 20.8 days with a range of 2 to 90 days. All of the patients underwent spinal instrumentation under fluoroscopic guidance. Of the 70 cases, 45 patients underwent single-level fixations while 24 patients underwent two-level spinal fixations. One patient underwent anterior odontoid screw placement for odontoid fracture type II (-Fig. 4).
In-hospital outcome and mortality: The total duration of hospital stay ranged from 8 to 90 days (mean of 21.4 days). During the hospital stay, 6 out of 70 patients expired (8.5\%). All of these were cervical spinal injury patients with poor respiratory function at admission. There was no in-hospital mortality for cases of dorsal or lumbar spinal injury. There was one mortality at 6 months' follow-up in a case of dorsal spinal injury, and its exact cause is unknown. Of all the expired cases, four of them were ASIA grade A, one was ASIA $B$, and the remaining two were ASIA C (-Table 1). On the basis of age groups, there was no mortality in group 1 , six in group 2 and one in group 3.

Follow-up: At the time of discharge, out of the 64 alive patients, 27 patients were ambulatory, 5 patients were 


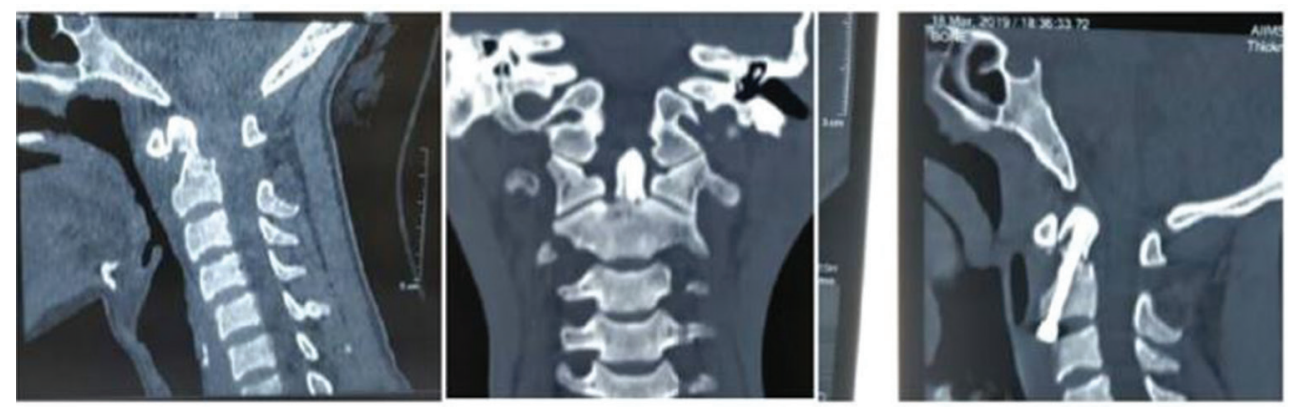

Fig. 4 Anterior odontoid screw placement for a type II odontoid fracture.

Table 1 Correlation of neurological outcome with ASIA grade at admission

\begin{tabular}{|l|l|l|l|l|l|}
\hline ASIA grade & Improved & Same & Worsened & Expired & Total \\
\hline A & 1 & 14 & 0 & 4 & 19 \\
\hline B & 2 & 6 & 0 & 1 & 9 \\
\hline C & 6 & 5 & 1 & 2 & 14 \\
\hline D & 0 & 15 & 0 & 0 & 15 \\
\hline E & 0 & 13 & 0 & 0 & 13 \\
\hline
\end{tabular}

Abbreviation: ASIA, American Spinal Injury Association.

Note: No mention of expired, worsened.

ambulatory with support, while 32 patients were dependent. In the follow-up period, nine patients showed neurological improvement (17.3\%), one patient deteriorated neurologically, one patient expired at 6 months' follow-up, and the remaining were in same neurological state as at discharge. Most of the patients who showed improvement had incomplete SCI at presentation (- Table $\mathbf{1}$ ).

\section{Discussion}

In our study, out of 70 patients, 45 were males and 25 were females with a male to female ratio of $1.8: 1$. This is comparable with previous studies in which males constituted 55 to $65 \%$ of all traumatic spine injury cases. ${ }^{3-6}$ In the present study, fall from height was the mode of injury in $77.1 \%$ of the cases followed by road traffic accidents in $22.9 \%$. This pattern is different from that in the western countries where road traffic accidents are a major cause of SCIs. According to a study by Donovan, ${ }^{7}$ motor vehicle accidents are a leading cause of SCI in the developed countries. According to various studies from India by Chacko et al, Maharaj, Singh et al, and Rathore et al, fall constitute most of the cases of spinal injury. ${ }^{8,3}$ These findings from the Indian studies corroborated with the findings in our study. Also in our case, most of the population was from hilly terrain, where fall from height constitutes a major cause of injuries in general.

Most of the patients sustaining spinal injuries are in the productive age group, which leads to physical, mental, and financial burden for individuals and their dependents. The mean age of patients in our study was 36 years (range: 16-65 years). A previous study published from Delhi, India, showed that among patients with spinal injuries, $40.9 \%$ were in their third decade. ${ }^{9}$
In our study, cervical cord involvement was seen in $28.5 \%$ cases, $32.9 \%$ involved dorsal spine, and 38.6\% involved the lumbar spine. On comparing the site of involvement with age groups, lumbar involvement was seen more commonly in the younger age group. Around $27.1 \%$ of our patients were ASIA grade $A$ at presentation whereas $18.6 \%$ were neurologically intact (ASIA E). In the age group analysis, we found that there was higher incidence of complete neurological injury in middle-age group (63.15\%), as compared with older age group (5.2\%). Our results seem to correlate with those results published by Muzumdar et al. ${ }^{10}$ They stated that complete neurological injuries were more commonly associated with younger age group.

In our study, burst fracture was the most common morphology of injury (60\%), followed by translational injuries (27.1\%). Most of our patients were operated after a mean of 20.8 days following their injury with a range of 2 to 90 days with the exception of one patient who presented late at almost 1 year after the injury. The long duration from the time of injury to the time of surgery can be attributed to the difficult terrain of the area and difficulties faced by the family in bringing the patient to the hospital. In addition, being a new institute with limited operation room time, the patients sometimes have to wait for surgery even after presenting to the hospital.

Our results show that all of the mortality was in cervical spinal injury patients and most of those were complete SCIs. Nine of our cases (12.8\%) showed neurological improvement on follow-up and rest of the patients remained same as preoperative status neurologically, except for one case in which there was neurological deterioration. Vale et al observed that $33 \%$ of patients with a complete thoracic SCI improved at least one ASIA grade. ${ }^{11}$ Bohlman and Freehafer have reported that neurologic recovery may occur if surgical decompression 
is performed within 2 years after the injury. ${ }^{12}$ In a study by Mahapatra et al, it was shown that surgical instrumentation was effective in complete injury as it facilitates early mobilization, chest physiotherapy, and reduced time spent in bed. ${ }^{13}$ In our study, it was seen that almost $42 \%$ cases of ASIA C improved on follow-up, suggesting that an incomplete SCI has chance of recovery.

\section{Conclusion}

As young people are most commonly affected, $\mathrm{SCI}$ has devastating effect on the patient and his/her family. Falls being the major cause of SCIs, appropriate preventive measures should be taken to prevent spinal injuries. Surgical management facilitates early mobilization and helps in rehabilitation of these patients. Neurological status at admission (ASIA grade) and level of injury remain the most important predictor of the outcome in patients of spinal trauma.

\section{Conflict of interest}

None declared.

\section{References}

1 Mathur N, Jain S, Kumar N, Srivastava A, Purohit N, Patni A. Spinal cord injury: scenario in an Indian state. Spinal Cord 2015;53(5):349-352

2 Srivastava RN, Singh A, Garg RK, Agarwal A, Raj S. Epidemiology of traumatic spinal cord injury: a SAARC perspective. Int J Biochem Mol Biol 2015;3:9-22
3 Parsons KC, Lammertse DP. Rehabilitation in spinal cord disorders. 1. Epidemiology, prevention, and system of care of spinal cord disorders. Arch Phys Med Rehabil 1991;72(4-S): S293-S294

4 Lan C, Lai JS, Chang KH, Jean YC, Lien IN. Traumatic spinal cord injuries in the rural region of Taiwan: an epidemiological study in Hualien county, 1986-1990. Paraplegia 1993;31(6):398-403

5 Hoque MF, Grangeon C, Reed K. Spinal cord lesions in Bangladesh: an epidemiological study 1994-1995. Spinal Cord 1999;37(12):858-861

6 Chacko V, Joseph B, Mohanty SP, Jacob T. Management of spinal cord injury in a general hospital in rural India. Paraplegia 1986;24(5):330-335

7 Donovan WH. Donald Munro lecture. Spinal cord injury-past, present, and future. J Spinal Cord Med 2007;30(2):85-100

8 Singh R, Sharma SC, Mittal R, Sharma A. Traumatic spinal cord injuries in Haryana: an epidemiological study. Indian J Community Med 2003;28(4):184-186

9 Upendra B, Mahesh B, Sharma L, et al. Correlation of outcome measures with epidemiological factors in thoracolumbar spinal trauma. Indian J Orthop 2007;41(4):290-294

10 Muzumdar D, Ventureyra ECG. Spinal cord injuries in children. J Pediatr Neurosci 2006;1:43-48

11 Vale FL, Burns J, Jackson AB, Hadley MN. Combined medical and surgical treatment after acute spinal cord injury: results of a prospective pilot study to assess the merits of aggressive medical resuscitation and blood pressure management. J Neurosurg 1997;87(2):239-246

12 Bohlman HH, Freehafer A. Late anterior decompression of spinal cord injuries. J Bone Joint Surg Am 1975;57:10-25

13 Siddhartha SS, Deepak G, Mahapatra AK. Traumatic paraplegia outcome study at an apex trauma centre. Indian J Neurotrauma 2011;8(1):33-36 